\title{
Un bibliorato de manuscritos teatrales del Siglo de Oro
}

\author{
Víctor INFANTES
}

(Universidad Complutense de Madrid)

\section{Resumen}

Se da noticia en este trabajo de un conjunto de 12 piezas teatrales manuscritas de la segunda mitad del siglo XVII, entre 1640 y 1660, fundamentalmente compuesto de entremeses y comedias. Se incluye una presentación general del testimonio, junto a la descripción codicológica individual de las piezas, con las correspondientes referencias bibliográficas referidas a cada una de ellas.

Palabras clave: Teatro; Manuscritos dramáticos; Siglo XVII.

\section{A folder of theatrical manuscripts of the Seventeenth Century}

\section{Abstract}

The author describes twelve manuscript plays, mainly entremeses (short interludes) and comedies, written in the second half of the seventeenth century, between 1640 and 1660. An overview of the collection is included, along with individual codicological descriptions and information on other copies.

Keywords: Drama; Manuscripts; Plays; Seventeenth Century. 
Tengo en mis manos una carpeta - verbi gratia 'bibliorato', con (o sin) permiso de la RAE - con doce obras manuscritas de cierta enjundia dramática. Bien sabemos que existe una notable cantidad de manuscritos teatrales áureos conocidos en la actualidad, los hay de comedias, entremeses, loas, bailes, jácaras, y demás etcéteras genéricas; hay catálogos de los fondos más singulares y numerosos donde se cuentan por cientos las piezas conservadas, pero siempre habrá más testimonios esperando la luz de su localización y descripción. Necesariamente hay autógrafos de sus autores (con sus diferentes estados de redacción), copias para los amigos, para los actores, para los aficionados, más copias para pasar censuras y licencias, para formar un repertorio con vistas comerciales; todas ellas en competencia con los impresos, las antologías editoriales, las colecciones del autor consagrado, las partes de los mejores ingenios, en conjunto, con el vasto negocio de la edición dramática para lectores que no vieron (o no verían jamás) las obras representadas en las tablas. Si el siglo XVI ya dio síntomas suficientes de la importancia de esta parcela literaria, el siglo siguiente confirmó con creces el número de ediciones y manuscritos, ocupando un amplio espacio comercial en competencia lectora con la poesía y la prosa áurea.

Este atadillo rescatado del olvido recoge doce obras manuscritas que, desde luego, no van a cambiar la historia de nuestro teatro barroco, pero que a buen seguro añaden varios testimonios de consideración a la hora de fijar los textos que contienen -algunos de notable importancia- y, claro está, que no aparece todos los días una docena de piezas teatrales manuscritas del siglo XVII con obras de Calderón de la Barca, Quiñones de Benavente o Pérez de Montalbán y algún que otro texto inédito o desconocido. El conjunto vale su peso dramático en algunos euros y en mucha literatura.

El testimonio que ha llegado hasta nosotros se trata de un conjunto de doce manuscritos independientes, todos en tamaño $4^{\circ}$ (entre 210/240x150/160 mm), en general copiados en pliegos de papel cosidos $-\mathrm{y}$ en algún caso sueltos-, la mayoría en aceptable estado de conservación; protegidos por dos tapas mudas de cartón de diferente medida, una de papel marmolado y otra de papel tintado, de finales del siglo XIX, simplemente sujetas horizontalmente por un cordón bicolor. La que hace las veces de cubierta ostenta una etiqueta de papel blanco donde figura anotado a mano en

Titivillus, ISSN 2387-0915, ISSN-e 2603-9966, 1 (2015), pp. 457-464 
tinta roja, letra del siglo XX: «ENTREMESES y COMEDIAS / Manuscritos del siglo XVII», con dos pequeños subrayados mediales; debajo, otra letra más antigua, siglo XIX, a lápiz, señala escuetamente: «Comedias». La mayoría son copias cuidadas de las obras, con letras, en general, de fácil lectura, aunque en algún caso hay correcciones y enmiendas, números 4 y 5 ; son todas del siglo XVII y, en general, a tenor de sus autores y cronología, les hemos asignado la «segunda mitad» del siglo, sin entrar en mayores precisiones caligráficas, probablemente escritas en el transcurso de un par de décadas: 1640-1660. No obstante, un grupo de piezas, números $3,6,7$ y 8 , llevan añadidas al finalizar el texto aprobaciones para su representación, con fechas de 1662, 1684-1690, 1682 y 1645, respectivamente; quizás ello indica que pertenecieran a un representante de comedias o al director de una compañía teatral, que formaba (o guardaba) un repertorio de obras destinado a su puesta en escena. Las foliaciones antiguas en alguna de ellas indican su inclusión en volúmenes facticios, números 1, 3, 5 y 7 , de los que ha sido separadas.

Sin ningún dato de procedencia, nada se puede aventurar de su origen más allá de quien preservó la colección con dos cubiertas de cartón barato y quien (o quienes) así la mantuvo hasta su reciente aparición en este venturoso año quijotesco y teresiano; no hay un nombre al que asignar su pertenencia ni ninguna mención que permita una identificación provisional. Es lo que hay.

Vaya, pues, su descripción sin grandes precisiones codicológicas - que tampoco necesitan-, con el (caprichoso) orden en que se encuentran, añadiendo las referencias bibliográficas esenciales de sus vinculaciones literarias -que recogen las anteriores y, por tanto, no ha lugar a repetirlas, salvo indicación expresa entre paréntesis (google, siempre aparte), dejando constancia de nuestro agradecimiento por habernos proporcionado los datos que se recogen en CATENTR $-{ }^{1}$ con los testimonios que conocemos de las obras, sin entrar en ningún caso en cuestiones textuales de dependencias o variantes y menos todavía en el complejo análisis de las atribuciones autoriales del teatro áureo. ${ }^{2}$

[1]) Luis Quiñones de Benavente, Entremés de los pareceres

Entremes Delos Pareceres / devenavente

\footnotetext{
${ }^{1}$ La sigla corresponde al Catálogo de entremeses de los Siglos de Oro [base de datos en prueba, cuyo responsable es Abraham Madroñal Maestro, cf. Abraham.Madronal@unige.ch].

2 Dada la (necesaria) repetición de las referencias bibliográficas que utilizamos, que tampoco son demasiadas, damos en la primera aparición la cita completa y remitimos con posterioridad a su mención abreviada; a cambio, cuando un nombre no lleva aparejada su referencia bibliográfica es porque se encuentra ampliamente citada (y comentada) en la cita principal, lo que nos ahorra su innecesaria repetición.
} 
$4^{\mathrm{o}}(230 \times 151 \mathrm{~mm}), 4 \mathrm{hs}$., numeración antigua en la esquina superior derecha del recto: «138-142», letra de la segunda mitad del siglo XVII; sin coser, buen estado de conservación.

Simón Palmer, ${ }^{3}$ I, p. 618, recoge una copia del siglo XIX del manuscrito Osuna en la Biblioteca del Instituto del Teatro de Barcelona, sobre este manuscrito Cotarelo y Mori ${ }^{4}$ realizó su edición, n. ${ }^{\circ}$ 294, II, pp. 696-700, recordando que «no ha pasado a la Biblioteca Nacional [de España]», hoy no localizado; Urzáiz Tortajada, ${ }^{5}$ II, p. 538, solo cita el manuscrito de Barcelona. Testimonio único en la actualidad, que tal vez sea el del fondo Osuna, aunque no aparece registrado en Rocamora; ${ }^{6}$ CATENTR.

\section{[2]) Mojiganga del camino al Pardo, que se bizo en el Auto de lo que va el hombre a}

Dios

Mojiganga delcamino Al pardo / quesehizo enelAuto deloqueba delhombre Adios [adorno caligráfico]

$4^{\circ}(240 \times 157 \mathrm{~mm}), 6$ hs., primera y última a modo de cubierta, las 4 hojas interiores con el texto, letra de la segunda mitad del siglo XVII, anotación posterior en primera hoja, recto, a tinta: «C 35»; cosido, buen estado de conservación.

URZÁIZ TORTAJADA, I, p. 199, sólo registra el auto calderoniano Lo que va del hombre a Dios, representado con el entremés El mayorazgo, también de Calderón de la Barca, en 1659, sin mención de ninguna mojiganga; CATENTR aporta un manuscrito de la Biblioteca Estense de Módena de los siglos XVII-XVIII, que indica se trata de una parodia de Ser fino y no parecerlo.

\section{[3]) ¿Antonio Cuervo?, Entremés de la Honrada}

Entremes De La Honrrada

$4^{\circ}$ (217x157 mm), 6 hs., primera y última a modo de cubierta, las 4 hojas interiores con el texto, numeración antigua en la esquina superior derecha del recto: «46-[49]», letra de la segunda mitad del siglo XVII, primera hoja, recto,

\footnotetext{
3 María del Carmen Simón PaLmer, Manuscritos dramáticos del Siglo de Oro de la Biblioteca del Instituto del Teatro de Barcelona, Madrid, CSIC (Cuadernos Bibliográficos, 34), 1977.

4 Emilio COTARelo y MORI, Colección de entremeses, loas, bailes, jácaras y mojigangas desde finales del siglo XVI a mediados del XVIII, Madrid, NBAE, 1911, 2 ts.; edición facsímile de José Luis Suárez García y Abraham Madroñal Durán, Granada, Universidad de Granada, 2000.

5 Héctor URZÁIz TORTAJADA, Catálogo de autores teatrales del siglo XVII, Madrid, FUE, 2002, 2 ts.

6 José María Rocamora, Catálogo abreviado de manuscritos de la Biblioteca del Excmo. Señor Duque de Osuna e Infantado, Madrid, Imprenta de Fortanet, 1882.
}

Titivillus, ISSN 2387-0915, ISSN-e 2603-9966, 1 (2015), pp. 457-464 
antes del título «.19», debajo del título, otra letra de época: «Lofirme en26 de / este mes de enero laño P[resen]te de / 1662 años / Joan Prozende [rúbrica]» y debajo, otra letra de época: «y lo firme / Entre», última hoja, verso, letra de época con anotaciones de cuentas; cosido, aceptable estado de conservación, con restos de humedad y hojas 2 y 3 con rasgadura central.

Urzáiz Tortajada (La Barrera, Simón Díaz, Lobato, etc.), I, p. 279, registra el manuscrito autógrafo de la Biblioteca Nacional de España, fondo Osuna (ROCAMORA, n. ${ }^{\circ}$ 663), fechado el 31 de mayo de 1659 a nombre de Antonio Cuervo; en la Biblioteca del Instituto del Teatro de Barcelona se conservan dos manuscritos, uno del siglo XVII, anónimo, y una copia del de la Biblioteca Nacional de España; SIMÓN PALMER, I, n. ${ }^{\circ}$ 603, este último lleva notas de Aureliano Fernández Guerra, que menciona un manuscrito de su propiedad, más antiguo y más completo, donde se afirma la autoría de Luis Quiñones de Benavente, de donde conjetura que «Cuervo» sería una errata - por j«cornejo»!- o un copista posterior; con el nombre de Doña Mata se imprimió a nombre de Calderón de la Barca (URZÁIZ TORTAJADA, I, p. 279). CATENTR.

\section{[4]) Entremés La paga del mundo}

Entremes Cantado / La paga delmundo / Representole / Antonio deprado

$4^{\circ}$ (216x157 mm), 6 hs., letra de la segunda mitad del siglo XVII; cosido, buen estado de conservación.

URZÁIZ TORTAJADA, II, p. 538, menciona un manuscrito del siglo XVIII de la Fundación Lázaro Galdiano, aunque confirma que se trata del baile de El mundo de Luis Quiñones de Benavente, con atribución añadida a este autor, incluido en su Jocoseria de 1645, recoge la cita de Buezo que lo cree mojiganga y supone que debió ser representada hacia 1630; CATENTRE señala el manuscrito Siqueira del siglo XVII (Estepa, ${ }^{7}$ pp. 203-210), como baile y con mezcla de fragmentos de otra obra titulada ElMartinillo, 1. ${ }^{a}$ parte.

\section{[5]) Pedro Calderón de la Barca, Comedia del Alma causa dos efectos}

Lagran Comedia delAlma causa dos efetos / de D. P[edr]o. Calderon

$4^{\circ}(217 \times 156 \mathrm{~mm}), 27 \mathrm{hs}$., numeración antigua en la esquina superior derecha del recto: «1-20», dos letras de la segunda mitad del siglo XVII; cosidas las veinte primeras hojas, aceptable estado de conservación, con bordes devastados en últimas hojas.

\footnotetext{
7 Luis ESTEPA, Teatro breve y de carnaval en el Madrid de los siglos XVII y XVIII. Estudios sobre los géneros dramáticos del baile y la folla, Madrid, Consejería de Educación y Cultura, 1994.
} 
URZÁIZ TORTAJADA, I, p. 184, menciona la representación en la Montería sevillana por la compañía de Bartolomé Romero en 1642 y su posterior impresión en 1682 a nombre de Calderón; también aporta la referencia de su inclusión, como anónima y con el título de El amor hace discretos, en 1671. ROCAMORA, n. ${ }^{\circ} 477$, menciona un manuscrito con este título en el fondo Osuna, hoy no localizado.

\section{[6]) Juan Pérez de Montalbán, El divino portugués San Antonio de Padua}

El di bino portugués S[an]. Antonio de / Padua / de Montalvan

$4^{\circ}(220 \times 152 \mathrm{~mm}), 52$ hs., dos primeras y dos últimas, en otro papel, a modo de cubiertas, las $48 \mathrm{hs}$. interiores con el texto, varias letras de la segunda mitad del siglo XVII; primera hoja, recto: «el di bino portugués S[an]. Antonio de / Padua $=$ », debajo anotación manuscrita posterior: «A 77» y debajo: «Expurgada y Atajado todo lo que se puede / escusar.= Con todas las censuras YLiz[encías].», segunda hoja, recto: «Mad[rid]. 13 de feb[rer]o de 1690 / Hagase la Comedia de s[a]n Ant[oni]o, ob / serbando loseñalado», en h. 48, recto, anotación manuscrita de época, aprobación de Pedro Francisco Lanina Sagredos para que se represente en Madrid en 6 de ¿octubre? 1684 y en el vuelto, otra aprobación manuscrita de época de Juan de Rueda y Cuebas de 25 de diciembre de 1684; cosido completo, con cosido interior en tres cuadernos, aceptable estado de conservación, con bordes devastados.

URZÁIZ TORTAJADA, II, p. 508, recoge la impresión de la obra en 1638 y su (probable) escritura en 1621, con una versión posterior; menciona la existencia de un manuscrito fechado en 1623, en la Biblioteca Nacional de España, donde se atribuye a «Don Bernardino de Obregón» (URZÁIZ TORTAJADA, I, p. 486, con extensa nota), que se considera, según Dixon, seudónimo de Pérez de Montalbán; existe otra obra del mismo título de Antonio Fajardo y Acevedo (URZÁIZ TORTAJADA, I, pp. 309-310). ROCAMORA, n. ${ }^{\circ} 1186$, menciona un manuscrito con este título en el fondo Osuna, hoy no localizado.

\section{[7]) Miguel de Valenzuela, Entremés del estudiante}

En[tremes] del estudiante

$4^{\circ}(214 \times 154 \mathrm{~mm}), 7$ hs., la primera y última, en otro papel, a modo de cubiertas, las 5 hojas interiores con el texto, numeración antigua en la esquina superior derecha del recto: «[1]-5», letra de la segunda mitad del siglo XVII, en h. 5 recto mención como autor de Miguel de Valenzuela (letra diferente de la que se encuentra en pieza n. ${ }^{\circ}$ 9), en h. 5 , vuelto, tres aprobaciones manuscrita del Maestro fray Juan Bautista Palacio, Calificador del Santo Oficio de 16 de diciembre de 1682 en Málaga, del Licenciado Gallego de 20 de diciembre de 1682 en Antequera y de Agustín de Tejada de 22 de diciembre de 1682 en

Titivillus, ISSN 2387-0915, ISSN-e 2603-9966, 1 (2015), pp. 457-464 
Antequera; cosido, en aceptable estado de conservación, primera hoja de guarda, en blanco, recortada.

URZÁIZ TORTAJADA, II, p. 535, recoge dos atribuciones, a Luis Quiñones de Benavente (Cotarelo) y a Tirso de Molina (Ríos), como anónimo fue publicado en 1658 en el Teatro poético y fue imitado por Luis Vélez de Guevara en La burla más sazonada (Cotarelo). CATENTR, recoge dos copias manuscritas del siglo XIX, en la Biblioteca del Instituto del Teatro de Barcelona y en The Hispanic Society of America, esta última con atribución a Tirso de Molina. Único testimonio manuscrito antiguo conocido.

\section{[8]) ¿Antonio Martínez de Meneses?, Entremés del heredero melindroso}

Entremes Del heredero melindroso

$4^{\circ}(210 \times 160 \mathrm{~mm}), 5$ hs., numeración en la esquina superior derecha del recto: «21-25», letra de la segunda mitad del siglo XVII, en h. 5, mención como autor a Antonio Martínez de Meneses y en la misma hoja, recto/vuelto, tras la cita de «Veala el censor», aprobación manuscrita de Juan Navarro de Espinosa de 4 de diciembre de 1645 en Madrid; sin coser, en aceptable estado de conservación.

No se recoge entre las obras de este autor (URZÁIZ TORTAJADA, II, pp. 423-424). No citado en ningún repertorio.

\section{[9]) Miguel de Valenzuela, Entremés de la chupona}

Entremes famoso dela / Chupona

$4^{\circ}$ (218x160 mm), 4 hs., letra de la segunda mitad del siglo XVII, en h. 4, recto, mención como autor de Miguel de Valenzuela (letra diferente de la que se encuentra en pieza n. ${ }^{\circ}$ 7) y en h. 4 recto/vuelto, aprobación manuscrita de época sin firmar; sin coser, buen estado de conservación.

Autor y obra no citados en ningún repertorio.

[10]) Fernando de Valenzuela, Contra el Amor Desengaño o Apolo y Leucotoe

Contra el Amor Desengaño / o / Apolo, y Leucotoe. / Fiesta Real de Zarzuela, con su Loa, / Saynete, y fin de Fiesta

$4^{\circ}(217 \times 160 \mathrm{~mm}), 20$ hs., letra de la segunda mitad del siglo XVII; cosido, buen estado de conservación.

En h. 1, recto lleva dos anotaciones de época con letras distinta a la de la copia, antes del título: «Calderon» y al margen del final del título: «Esta Zarzuela / la escriuio D. P.॰ / Calderon, y por / hauerse hallado / entre los pape- / les de Valen- / zuela juzga- / ron ser suya y la copiaron dándole por autor della.». 
URZÁIZ TORTAJADA, I, p. 184, con atribución a Calderón de la Barca en uno de los manuscritos de la Biblioteca Nacional de España, que conserva otros dos; es dudosa la autoría, aunque existen partituras musicales para esta obra de Juan Hidalgo (Stein), colaborador en otras obras calderonianas.

[11]) ¿Marcelo Antonio de Ayala y Guzmán?, Guerras de amory de celos

Guerras de Amor y de Zelos

$4^{\circ}(215 \times 156 \mathrm{~mm}), 26 \mathrm{hs}$., letra de la segunda mitad del siglo XVII; sin coser, buen estado de conservación.

URZÁIZ TORTAJADA, I, p. 148, ofrece un excelente resumen de la complicada atribución a este dramaturgo, a partir de un manuscrito de la Biblioteca Nacional de Madrid con la fecha de su representación en Palacio el 6 de noviembre de 1687 y la publicación de una suelta en Granada, s. a.; también se imprimió a nombre de Matías de Ayala y se ha atribuido a Luis de Guzmán (Medel y García de la Huerta) y a Matías de Guzmán (La Barrera).

[12]) ¿Antonio de Solís y Rivadeneyra?, Entremés de los Buñuelos

Entremes, delos Vuñuelos, / enmendado, yañadido

$4^{\circ}$ (215x154 mm), 5 hs., letra de la segunda mitad del siglo XVII, sin coser, buen estado de conservación.

URZÁIZ TORTAJADA, II, p. 612, recoge esta atribución en un ejemplar de su comedia Un bobo hace ciento de la Biblioteca Nacional de Madrid; también lo da como anónimo en una impresión de 1691 de la Arcadia de entremeses y en una suelta de 1792 (URZÁIZ TORTAJADA, I, p. 66); existe también un baile con el mismo título (URZÁIZ TORTAJADA, I, p. 66). ROCAMORA, n. ${ }^{\circ} 350$ menciona un manuscrito con este título del fondo Osuna, hoy no localizado. CATENTR recoge tres manuscritos tardíos, dos en la Biblioteca Nacional de España y uno del siglo XIX en la Biblioteca del Instituto del Teatro de Barcelona, copia de la Arcadia de entremeses (SIMÓN PALMER, I, p. 408), así como diferentes ediciones como suelta de los siglos XVIII y XIX.

Titivillus, ISSN 2387-0915, ISSN-e 2603-9966, 1 (2015), pp. 457-464 